\title{
Revisión de estudios funcionales en experimentación básica en Urología
}

\author{
Bustamante Alarma S. \\ Servicio de Urología. Hospital Universitario Puerta de Hierro. Madrid
}

Actas Urol Esp. 2008;32(3):390-395

\begin{abstract}
RESUMEN
REVISIÓN DE ESTUDIOS FUNCIONALES EN EXPERIMENTACIÓN BÁSICA EN UROLOGÍA

La finalidad de este trabajo de revisión, tiene por objeto, dar a conocer a las sociedades médicas y concretamente a las urológicas de habla hispana, el resultado del trabajo desarrollado durante estos últimos 25 años, a nivel del Sistema Urinario, por parte, de un grupo de investigadores del Departamento de Fisiología de la Facultad de Farmacia de la Universidad Complutense de Madrid (UCM) dirigido por el catedrático Albino García Sacristán, que ha proporcionado un considerable número de publicaciones en revistas internacionales con un índice de impacto relevante.

$\mathrm{El}$ autor de este trabajo, ha colaborado en distintos proyectos, con la realización de su tesis doctoral y continúa en la actualidad.

Palabras clave: Experimentación básica. Uréter. Vejiga. Arterias peneanas y arterias de la próstata.
\end{abstract}

\section{ABSTRACT}

REVIEW OF FUNCTIONAL STUDIES IN BASIC EXPERIMENT IN UROLOGY

The purpose of this review work, has as a goal, make known to the medical societies and concretely to the urologic Spanish-speaking, the result of the work developed during these last 25 years, at level of the Urinary System, by part, of a group of researchers of the Physiology Department of the Pharmacy Faculty of the University Complutense of Madrd (UCM) directed by professor Albino García Sacristán, that it has provided a considerable number of publications in international magazines with a relevant impact index.

The author of this work, it has collaborated in different projects, with the accomplishment of your doctoral thesis and continues at present.

Keywords: Basic experiment. Ureter. Bladder. Arteries penis and arteries of the prostate.

$\mathrm{L}^{\infty}$ os estudios experimentales funcionales en baños de órganos, reproducen fielmente el comportamiento de las estructuras, como si se estas, se encontrasen en su medio natural. Para ello es necesario trabajar con ellas, en unas condiciones las más idóneas posibles, es decir, recién obtenidas en el matadero municipal y transportadas en un medio nutricio adecuado y con una $\mathrm{T}^{\mathrm{o}}$ correcta. En estas condiciones, se proporciona a las preparaciones, una tensión previamente establecida.

Se inicia el estudio, al incubarse estas, con diferentes sustancias exógenas o al estímulo de las mismas, con estimulación eléctrica transmural (EET) a diferentes frecuencias. Es de resaltar, que una actividad fisiológica tan común, como la micción se debe en última instancia y fundamentalmente a la liberación de neurotransmisores desde terminales nerviosas pre-sinápticas, los cuales, se acoplan a sus receptores específicos en el músculo e inducen una respuesta ya sea de contracción ó de relajación. Si se analiza, el método de trabajo utilizado, se comprueba que existe un paralelismo significativo entre estos estudios funcionales en experimentales básica y los estudios urodinamicos 
convencionales y/o los de monitor ambulatorio que se realizan en nuestros gabinetes de Urodinámica. El autor de este trabajo, lo denomina, Urodinámica "in vitro" ó Urodinámica realizada en el laboratorio.

\section{TRABAJOS EN URÉTER Y VEJIGA URINARIA}

\section{Regulación adrenérgica}

El uréter es un conducto estrecho de músculo liso encargado de transportar el bolo de orina desde el riñón hasta la vejiga. Dicho transporte se inicia como consecuencia de señales eléctricas generadas desde un marcapasos de localización intrarrenal. A nivel del segmento distal ureteral existe una rica inervación autónoma adrenérgica, colinérgica, nitrérgica y péptidérgica constituyendo densos plexos neuro musculares, peri vascular y subepiteliales ${ }^{1-4}$. La existencia de esta rica inervación sugiere una implicación relevante de los nervios autónomos en el control de la actividad de la musculatura lisa uretral. Estudios funcionales in vitro ${ }^{5,6}$ en el uréter de perro y cerdo, respectivamente, indican que la noradrenalina favorece el transporte de orina a través del uréter al incrementar la actividad fásica y el tono del musculatura lisa ureteral, mientras la acetilcolina participa de forma más activa en el cierre ureteral, una vez evacuado el bolo de orina a la vejiga. Asimismo, diversos péptidos como el neuropéptido Y (NPY) y el polipéptido intestinal vaso activo (VIP) están involucrados en la modulación de la respuesta de la noradrenalina a través de la potenciación de ambos componentes contráctiles (fásico y tónico) ureterales ${ }^{3}$. Además, una regulación no adrenérgica, no colinérgica (NANC) ha sido puesta de manifiesto en la neurotransmisión inhibitoria ureteral. Así, el óxido nítrico $(\mathrm{NO})^{4}$ y el péptido relacionado con el gen de la calcitonina (CGRP) relajan la musculatura lisa ureteral a través de un mecanismo dependiente del guanosin 35 monofosfato $(\mathrm{GMPc})^{7}$, involucrando en la activación de canales de $\mathrm{K}^{+}$sensibles a la glibenclamida. Estudios in vitro han mostrado un control adrenérgico $^{8}$, colinérgico 9 e histaminérgico ${ }^{10}$ de dicha unidad funcional.

Estudios histoquímicos e inmunocitoquímicos revelan la existencia de una rica red de fibras nerviosas adrenérgicas en el uréter distal con un discurrir típicamente sinuoso formando densos plexos neuromusculares, peri vasculares y subepiteliales en el uréter del caballo ${ }^{1,12}$. La inervación adrenérgica era más rica en los trayectos finales del uréter (uréter intravesical) en comparación con las porciones superiores, las cuales, están inervadas por escasos y pequeños troncos nerviosos procedentes del plexo pélvico. Esta inervación adrenérgica podría desempeñar un papel fisiológico en la coordinación del uréter intravesical durante las fases de llenado y vaciado de la vejiga urinaria. En esta región no se han visualizado ganglios intramurales asociados en el uréter humano, pero sí se han demostrado células TH-IR en ganglios intramurales en el uréter del caballo, los cuales, podrían ejercer un control local de la perístasis ureteral ${ }^{12}$. Estudios funcionales in vitro en uréter del cerdo muestran que la noradrenalina estimula ambos componentes contráctiles (actividad fásica y tono) de la musculatura lisa ureteral. Dicha respuesta es mediada esencialmente a través de receptores adrenérgicos $\alpha_{1}$, aunque también receptores $\alpha_{2}$ estarían implicados en el mantenimiento del tono de la pared ureteral $^{5}$. La relajación de la pared ureteral del cerdo se llevaría a cabo a través de la estimulación de receptores $\beta_{1^{-}}$y $\beta_{2}$ adrenérgicos ${ }^{5}$.

\section{Regulación colinérgica}

Estudios histoquímicos revelan la presencia de una rica inervación de fibras acetilcolinesterasa positivas (AChE) formando densos plexos neuromusculares, subepiteliales y peri vasculares en el uréter intravesical del caballo ${ }^{2}$. Estudios in vitro revelan la existencia de una población heterogénea de receptores muscarínicos en el uréter intravesical del cerdo. Así un 83\% de dicha población pertenecen al subtipo $\mathrm{M}_{2}$ mientras que el $17 \%$ restante están formados por una mezcla de receptores $\mathrm{M}_{1}, \mathrm{M}_{3} \mathrm{y} \mathrm{M}_{4}{ }^{11}$. No obstante, la ACh provoca la contracción de la musculatura lisa ureteral del cerdo esencialmente a través de los receptores muscarínicos $\mathrm{M}_{3}$, desempeñando los otros 3 subtipos muscarínicos presentes un papel funcional menos relevante $^{6}$.

\section{Implicación del Oxido nítrico (NO) en el uréter}

Estudios histoquímicos e inmunocitoquímicos han mostrado la existencia de fibras NADPH-diaforasa positivas y NOS-IR en el porcino ${ }^{4}$ en forma de finas fibras localizadas en el músculo con trayectoria paralela al eje longitudinal de la fibra muscular, 
alrededor de vasos y por debajo del urotelio. Además, el NO liberado desde nervios ureterales en respuesta a estimulación eléctrica transmural y añadido exógenamente en forma de sal acidificada de $\mathrm{NaNO}_{2}$ relajan el uréter intravesical del cerdo ${ }^{4}$ siendo dicha respuesta relajante producida a través de un mecanismo dependiente del cGMP, el cual, favorece la apertura de canales de $\mathrm{K}^{+}$sensibles a la glibenclamida $^{7}$. Diferentes estudios han mostrado la implicación de la vía L-arginina/NO en la neurotransmisión inhibitoria del uréter ${ }^{4}$.

\section{Implicación del Neuropéptido Y (NPY) en la vejiga $y$ uréter}

Existe una rica distribución de fibras NPY-IR en la vejiga y el uréter intravesical del caballo ${ }^{3}$ localizadas a nivel de la adventicia, muscular y mucosa así como alrededor de las arterias de pequeño calibre. Asimismo, se ha descrito parte de la inervación NPY en ganglios intramurales asociados, lo cual, determina su origen intrínseco. El uréter del caballo ${ }^{3}$ está ricamente inervado por fibras NPY-IR. Sin embargo, el NPY no afecta la actividad fásica y el tono de la musculatura lisa ureteral $^{3}$. El NPY, sin embargo, sí incrementa la actividad fásica y el tono inducido por la noradrenalina en el uréter intravesical equino, siendo dicha respuesta mediada a través de receptores $\mathrm{Y}_{2}$ postsinápticos desempeñando, por tanto, un importante papel neuromodulador de la motilidad ureteral $^{3}$. Además, el NPY origina una potente acción vasoconstrictora de las arterias de resistencia ureterales a través de receptores $\mathrm{Y}_{1}$, lo cual, sugiere una regulación del flujo sanguíneo local ${ }^{3}$. Los efectos potenciadores del NPY son debidos a su acción en zonas postsinápticas que incluyen cambios en los mecanismos de transducción intracelular (incremento en el influjo o en la sensibilidad al $\left.\mathrm{Ca}^{2+}\right)^{13}$.

\section{Adenosina en uréter}

Además, en el uréter intravesical del cerdo, la adenosina está involucrada en la modulación de la neurotransmisión NANC excitatoria únicamente a través de un mecanismo postsináptico. Así, la adenosina formada a partir del ATP, bien liberado desde los nervios o añadido exógenamente, relaja el uréter a través de la activación de receptores $\mathrm{A}_{2 \mathrm{~B}}$ presentes en la musculatura lisa ${ }^{14}$.

\section{Taquicininas (TKs)}

Se trata de una familia de péptidos que incluyen sustancia P (SP) y neurocininas Ay B (NKA y NKB), relacionados estructuralmente y localizados en fibras aferentes primarias amielínicas tipo $\mathrm{C}$ sensible a capsaicina. En el sistema nervioso periférico la SP y la NKA son consideradas sustancias neurotransmisoras y/o neuromoduladoras. Así, la SP jugarian un papel como neurotransmisor sensorial y su liberación periférica provoca vaso dilatación, estableciéndose su implicación en la génesis de la denominada "inflamación neurógenica”, provocada como consecuencia de la permeabilidad vascular provocada por la liberación presente en fibras nerviosas y nos permitiría comprender la acciones "eferentes" de las fibras aferentes sensoriales. La acción de las taquicininas es mayoritariamente excitadora, no obstante, también induce una potente acción vasodilatador sobre el endotelio.

Las taquicininas interaccionan con tres tipos de receptores denominados: $\mathrm{NK}_{1}, \mathrm{NK}_{2}$ y $\mathrm{NK}_{3}$. Así, a nivel del Tracto Urinario participan en la modulación local de la motilidad ureteral, vesical y uretral, principalmente la SP y NKA a través de su unión con receptores $\mathrm{NK}_{2}$, favoreciendo la respuesta contráctil ${ }^{15,16}$. Además, en la actualidad existe evidencia anatómica, neuroquímica y fisiológica, que le atribuyen un papel importante en la regulación de la micción y en la medicación del dolor vesical. Así, la taquicininas podría desempeñar un papel más relevante como mediadores fisiopatológicos que como reguladores fisiológicos. La liberación de taquicininas desde los nervios sensoriales ocurre durante las cistitis y provee una contribución neurógenica a los procesos inflamatorios del TUI. De tal modo que el aumento prolongado en el tono vesical producido por acción de las taquicininas, contribuiría a generar sintomas de urgencia y frecuencia miccional durante la cistitis (Fig. 1).

\section{Péptido intestinal vasoactivo (VIP) y Péptido hipofisario activador de la adenilato ciclasa (PACAP).}

Recientes estudios han puesto en evidencia el efecto relajante a nivel del uréter intravesical del cerdo ${ }^{17} \mathrm{y}$ cuello vesical $^{18,19}$ inducido por VIP-PACAP (Fig. 2).

\section{TRABAJOS EN ARTERIAS PENEANAS}

\section{Arterias helicinas peneanas}

La línea de investigación llevada a cabo por el grupo de la Facultad de Farmacia de la UCM desde 


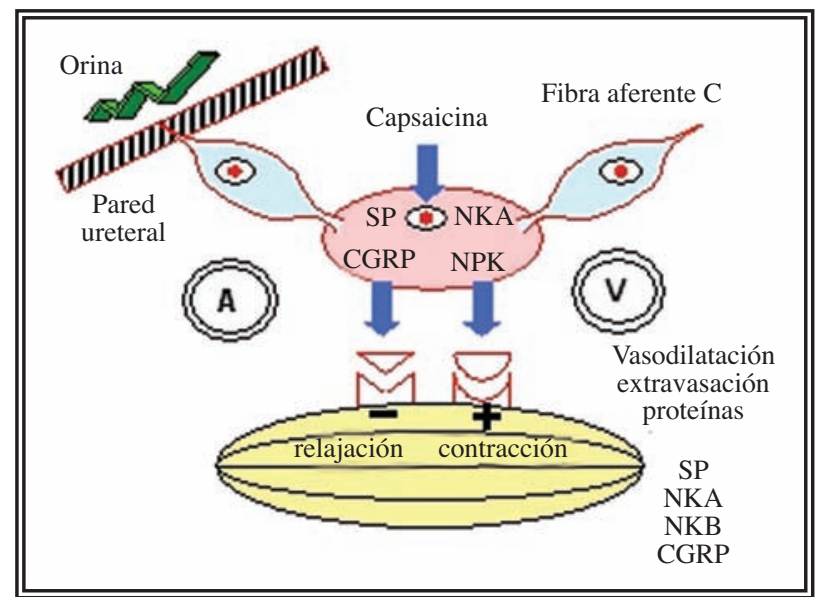

FIGURA 1. Representación esquemática de la implicación de las taquicininas, liberadas desde nervios sensoriales tipo $C y$ sus receptores especificos en la musculatura lisa ureteral.

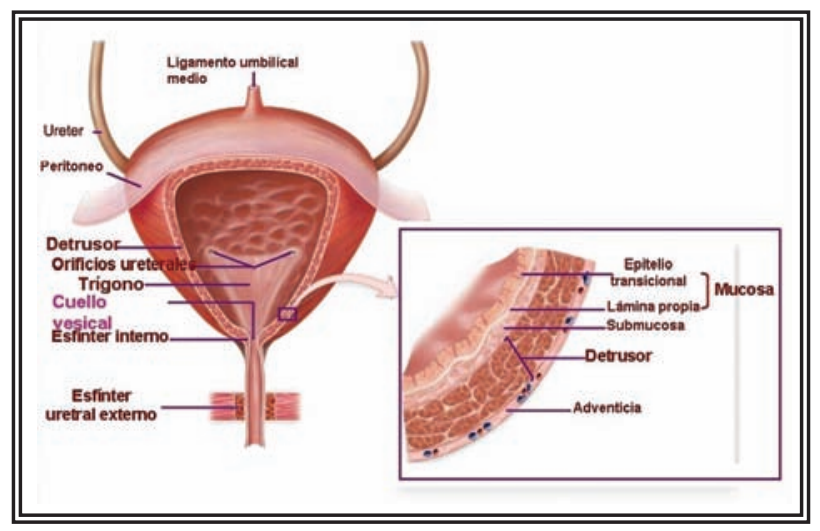

FIGURA 2. Representación de la zona utilizada para el estudio de cuello vesical.

sus inicios se ha centrado en el estudio de diversos aspectos del control nervioso y local del músculo liso visceral del tracto genitourinario y del músculo liso vascular. Se ha establecido un modelo experimental in vitro para el estudio de las características fisiofarmacológicas de las arterias de resistencia peneanas o arterias helicinas ${ }^{20}$, que actúan como esfínteres fisiológicos regulando el flujo sanguíneo entre la circulación sistémica y los sinusoides cavernosos. Dichas arterias poseen una inervación adrenérgica funcional, con receptores adrenérgicos $\alpha_{1} \mathrm{y}$ $\alpha_{2}$ postsinápticos que median los efectos contráctiles de la noradrenalina, y receptores inhibidores $\beta_{2}$ que median efectos relajantes ${ }^{21}$. Existen también receptores presinápticos $\alpha_{2}$ que inhiben la liberación de $\mathrm{NO}^{22}$. El NPY, cotransmisor junto con la noradrenalina en nervios peri vasculares simpáticos, actúa a través de una población heterogénea de receptores $Y_{1}$ e $Y_{2}$ que potencian las respuestas contráctiles de la noradrenalina, aunque también provoca vaso dilatación a través de receptores endoteliales de naturaleza desconocida ${ }^{23}$. Las arterias helicinas también poseen una densa inervación nitrérgica y el NO es el mediador de las respuestas inhibidoras NANC de estas arterias, y relaja el músculo liso arterial activando canales de $\mathrm{K}^{+}$activados por $\mathrm{Ca}^{2+}\left(\mathrm{K}_{\mathrm{Ca}}\right)^{24}$ al igual que ocurre con el tejido eréctil trabecular ${ }^{25}$. En un modelo de hipertensión renovascular, se ha demostrado un menor efecto vaso dilatador tanto neurógenica como dependiente de endotelio en las arterias pequeñas peneanas ${ }^{26}$. En cuanto al papel del endotelio vascular en la modulación de la reactividad de las arterias helicinas, las relajaciones dependientes del endotelio están mediadas por la liberación de NO y de un factor de naturaleza desconocida (EDHF) que actúa incrementando la conductancia de los canales $\mathrm{K}_{\mathrm{Ca}}$ y la bomba $\mathrm{Na}^{+}-\mathrm{K}^{+}$-Atpasa ${ }^{27}$.

$\mathrm{El}$ correcto funcionamiento del mecanismo venooclusivo parece esencial para el mantenimiento de la tumescencia peneana. La acetilcolina induce en la vena dorsal profunda del pene una relajación dependiente de endotelio a través de receptores muscarínicos del subtipo $\mathrm{M}_{3}{ }^{28}$, siendo el principal mecanismo utilizado la activación de la vía del NO/GMPc, que puede ser modulada por la apertura de canales de $\mathrm{K}_{\mathrm{V}} \mathrm{y}$ en menor medida por activación independiente de NO de canales $\mathrm{K}_{\mathrm{Ca}}$. La acetilcolina puede llegar a producir una contracción venosa si disminuye la disponibilidad de NO, como ocurre en enfermedades vasculares tipo diabetes o hipertensión; es entonces cuando se activarían los receptores muscarínicos endoteliales $M_{1}$ relacionados con la vía de la ciclo-oxigenasa ${ }^{29}$.

Por otra parte, el grupo de investigación ha desvelado recientemente aspectos relevantes de los mecanismos de acción en arterias de resistencia peneana de fármacos vaso activos utilizados en el tratamiento de la DE. Así, la eficacia clínica de la $\mathrm{PGE}_{1}$ y el sildenafilo está correlacionada con su capacidad de relajar las arterias helicinas in vitro. Mientras que la $\mathrm{PGE}_{1}$ incrementa los niveles de AMPc y activa canales $\mathrm{K}_{\mathrm{ATP}}$, la capacidad relajante de sildenafilo está basada en el incremento de los efectos del NO liberado de forma basal desde el endotelio vascular $\mathrm{y}$ activación de canales $\mathrm{K}_{\mathrm{Ca}}$; en ambos fármacos producen un activación cruzada de las vías de señalización mediadas por nuclelótidos ${ }^{30-34}$. 


\section{RECIENTES PUBLICACIONES EN CUELLO VESICAL}

Estudios morfológicos e inmunohistoquimicos han puesto en evidencia la implicación de los receptores $\alpha 2$ adrenérgicos en la regulación del cuello vesical. Sin embargo no se conoce el papel de los neurotransmisores que inducen un efecto relajante o inhibitorio sobre el mismo. Se sabe que tanto el péptido intestinal vaso activo (VIP) presente en fibras motoras, como el polipéptido hipofisario activador de la adenilato ciclasa (PACAP) en fibras sensibles inducen un efecto relajante a nivel del cuello de la vejiga del cerdo. Tanto VIP como las 2 isoformas de PACAP el 27 y el 38 hemos comprobado que relajan de una manera similar, lo cual sugiere que es un receptor VPAC. Además se ha visto que el antagonista de PACAP (6-38) únicamente produce efecto inhibitorio a altas concentraciones, lo cual sugiere que es un receptor VPAC2.

Desde fibras motoras se libera VIP que se une a este receptor, además existe un receptor (auto receptor de tipo inhibitorio) cuya activación modula la liberación de VIP (actúa como un sensor). Por otra parte desde fibras sensoriales existen receptores presinápticos faciltadores PACl que favorecen la liberación de NO induciendo un efecto relajante. El NO se difunde a través de la membrana celular (bicapa lípidica) y relaja a través del CMPC. Además, esta conjugación produce relajación con salida de $\mathrm{K}$ a través de la activación de canales de $\mathrm{K}$ voltaje dependiente hecho confirmado por que el uso de la 4 amino piridina (bloqueante específico de los canales de $\mathrm{K}$ dependientes de voltaje, hecho que no ocurre con el resto de canales de $\mathrm{K}^{35}$, 36. Se han realizado estudios intracelulares de musculatura lisa de vejiga urinaria en cobaya y la implicación de la contracción inducida por receptores muscarínicos.

\section{ESTUDIOS PRELIMINARES REALIZADOS EN ARTERIAS PROSTÁTICAS}

Está establecido por estudios previos, que enfermedades cardiovasculares, como la hipertensión y la arteriosclerosis, inducen una reducción del flujo sanguíneo periférico y esta hipoxia, puede representar un riesgo extrínseco que favorece la hiperplasia benigna de próstata (HBP). Otro hecho que se produce, como consecuencia de una deficiente vascularización prostática, es el debido a un aumento de la masa glandular y a una disminución de los niveles plasmáticos de testosterona. No existe información, sin embargo, sobre los mecanismos que involucran estos efectos, inducida por la testosterona sobre el flujo sanguíneo prostático. Están en estudio en la actualidad, en nuestro departamento mediante, técnicas inmunohistoquímicas y miógrafos micro vascular para el registro de fuerza isométrica, la función que desempeñan la neurotransmisión nitrérgica y peptidérgica. Así como, diferentes hormonas esteroideas sobre la reactividad vascular prostática, haciendo especial hincapié en aquellos agentes como son el péptido intestinal vasoactivo (VIP), el péptido hipofisiario activador de la adenilato ciclasa (PACAP), la testosterona y la dihidrotestosterona cuyos niveles plasmáticos y expresiones tisulares de sus receptores se encuentran modificados tanto en la HPB como en el cáncer de próstata.

\section{CONCLUSIÓN}

La metodología empleada en el desarrollo de esta línea de investigación es un ejemplo representativo del carácter traslacional que debe presidir la investigación en Urología en un momento de expansión y desarrollo farmacológico relevante para el tratamiento de múltiples patologías de la unidad funcional vésico-prostática. Por tanto, es necesario estimular el desarrollo de iniciativas de investigación en nuestra especialidad basadas en modelos en los que coexista cooperación entre profesionales, impulsando de esta manera, la investigación urológica traslacional.

\section{REFERENCIAS}

1. Prieto D, Benedito S, Rivera L, Hernández M. García-Sacristán A. Autonomic innervation of the equine urinary bladder. Anat Histol Embryol. 1990;19(3):276-285.

2. Prieto D, Simonsen U, Martin J, Hernández M, Rivera L, Lema I et al. Histochemical and functional evidence for a cholinergic innervation of the equine ureter. J. Auton Nerv Syst. 1994; 47(3): 159-170.

3. Prieto D, Hernández M, Rivera L, García-Sacristán A, Simonsen U. Distribution and functional effects of neuropeptide Y on equine ureteral smooth muscle and resistance arteries. Peptides. 1997;69(3):155-165.

4. Hernández M, Prieto D, Orensanz LM, Barahona MV, GarcíaSacristán A, Simonsen U. Nitric oxide is involved in the nonadrenergic, non- cholinergic inhibitory neurotransmission of the pig intravesical ureter. Neurosci Lett. 1995b;186(1):33-36.

5. Hernández M, Prieto D, Simonsen U, Rivera L, Barahona MV, García-Sacristán A. Noradrenaline modulates smooth muscle activity of the isolated intravesical ureter of the pig through different types of adrenoceptors. Br J Pharmacol. 1992;107(4): 924-931.

6. Hernández M, Simonsen U, Prieto D, Rivera L, García P, Ordaz $\mathrm{E}$, et al. Different muscarinic receptor subtypes mediating the phasic activity and basal tone of pig isolated intravesical ureter. Br J Pharmacol. 1993;110(4):1413-1420. 
7. Hernández M, Prieto D, Orensanz LM, Barahona MV, JiménezCidré M, Rivera L et al. Involvement of a glibenclamide-sensitive mechanism in the nitrergic neurotransmission of the pig intravesical ureter. Br J Pharmacol. 1997.120(4);509-516.

8. Rivera L, Hernández M, Benedito S, Prieto D, García-Sacristán A. Mediation of contraction and relaxation by alpha- and betaadrenoceptors in the ureterovesical junction of the sheep. Res Vet Sci. 1992a;52(1):57-61.

9. Rivera L, Hernández M, Benedito S, Prieto D, García-Sacristán A. Mediation of contraction by cholinergic receptors in the ureterovesical junction. J Auton Pharmacol. 1992b;12(3):75-181.

10. Benedito S, Prieto D, Rivera L, Costa G, García-Sacristán A. Mechanisms implicated in the histamine response of the sheep ureterovesical junction. J Urol. 1999;146(1):184-187.

11. Hernández M, García-Sacristán A, Orensanz LM. Muscarinic binding sites of the pig intravesical ureter. J Auton Pharmacol. 1995a; 15(5):351-359.

12. Prieto D, Hernández M, Rivera L, Ordaz E, García-Sacristán A. Catecholaminergic innervation of the equine ureter. Res Vet Sci. 1993;54(3):312-318.

13. Prieto D, Simonsen U, Nyborg NC. Regional involvement of an endothelium- derived contractile factor in the vasoactive actions of neuropeptide $\mathrm{Y}$ in bovine isolated retinal arteries. $\mathrm{Br}$ J Pharmacol. 1995. Nov; 116(6):2729-2737.

14. Hernández M, Barahona MV, Bustamante S, García-Sacristán A, Orensanz LM. A2B adenosine receptors mediate relaxation of the pig intravesical ureter: adenosine modulation of non adrenergic non cholinergic excitatory neurotransmission. $\mathrm{Br} \mathrm{J}$ Pharmacol. 1999;126(4):969-978.

15. Bustamante S, Orensanz LM, Barahona MV, Contreras J, García Sacristán A, Hernández M. Tachykininergic excitatory neurotransmission in the pig intravesical ureter. J Urol. 2000; 164(4): 1371-1375.

16. Bustamante S, Orensanz LM, Barahona MV, García Sacristán A, Hernández M. NK2 tachykinin receptors mediate contraction of the pig intravesical ureter: tachykinin-induced enhancement of non adrenergic non cholinergic neurotransmission. Neurourol Urodynam. 2001; 20(3):297-308.

17. Hernández M, Barahona MV, Recio P., Rivera L, Benedito S, Martínez AC et al. Heterogeneity of neuronal and smooth muscle receptors involved in the VIP- and PACAP-induced relaxations of the pig intravesical ureter. $\mathrm{Br} \mathrm{J}$ Pharmacol. 2004; 141(1):123-131.

18. Hernández M, Barahona MV, Recio, P, Benedito, S, Martínez, AC, Rivera, L, et al. Neuronal and smooth muscle receptors involved in the PACAP- and VIP-induced relaxations of the pig urinary bladder neck. Br J Pharmacol. 2006;149(1):100-109.

19. Hernández M, Barahona MV, Recio P, Bustamante S, Benedito $\mathrm{S}$, Rivera $\mathrm{L}$ et al. PACAP 38 is involved in the non adrenergic non cholinergic inhibitory neurotransmission in the pig urinary bladder neck. Neurourol Urodynam 2006; 25(5):490-497.

20. Simonsen U, Garcia-Sacristan A, Prieto D. Penile arteries and erection. J Vasc Res. 2002 Jul-Aug;39(4):283-303.

21. Simonsen U, Prieto D, Hernandez M, Saenz de Tejada I, GarciaSacristan A. Adrenoceptor-mediated regulation of the contractility in horse penile resistance arteries. J Vasc Res. 1997MarApr; 34(2):90-102.

22. Simonsen U, Prieto D, Hernandez M, Saenz de Tejada I, GarciaSacristan A. Prejunctional alpha 2-adrenoceptors inhibit nitrergic neurotransmission in horse penile resistance arteries. J Urol. 1997 Jun; 157(6):2356-2360.

23. Prieto D, Arcos LR, Martinez P, Benedito S, Garcia-Sacristan A, Hernandez M. Heterogeneity of the neuropeptide Y (NPY) contractile and relaxing receptors in horse penile small arteries. $\mathrm{Br}$ J Pharmacol. 2004 Dec;143(8):976-986.
24. Simonsen U, Prieto D, Saenz de Tejada I, Garcia-Sacristan A. Involvement of nitric oxide in the non-adrenergic non-cholinergic neurotransmission of horse deep penile arteries: role of charybdotoxin-sensitiveK(+)-channels. Br J Pharmacol. 1995 Nov; 116(6):2582-2590.

25. Recio P, Lopez PG, Hernandez M, Prieto D, Contreras J, GarciaSacristan A. Nitrergic relaxation of the horse corpus cavernosum. Role of cGMP. Eur J Pharmacol. 1998 Jun; 12:351(1):8594.

26. Martinez AC, Hernandez M, Prieto D, Raposo R, Pagan RM, Garcia-Sacristan A, et al. Enhanced histamine-mediated contraction of rabbit penile dorsal artery in diet-induced hypercholesterolemia. Vascul Pharmacol. 2006; Jan 44(1):34-41. Epub 2005 Nov 11.

27. Prieto D, Simonsen U, Hernandez M, Garcia-Sacristan A. Contribution of $\mathrm{K}+$ channels and ouabain-sensitive mechanisms to the endothelium-dependent relaxations of horse penile small arteries. Br J Pharmacol. 1998; Apr 123(8):1609-1620.

28. Martinez AC, Hernandez M, Rivera L, Recio P, Garcia-Sacristan A, Benedito S. Muscarinic receptor subtypes mediate vasorelaxation in isolated horse deep dorsal penile vein. Urology. 2003 Aug;62(2):357-361.

29. Martinez AC, Prieto D, Hernandez M, Rivera L, Recio P, GarciaSacristan A, et al. Endothelial mechanisms underlying responses to acetylcholine in the horse deep dorsal penile vein. Eur $\mathrm{J}$ Pharmacol. 2005 May 16;515(1-3):150-159.

30. Ruiz Rubio JL, Hernandez M, Rivera de los Arcos L, Martinez AC, Garcia-Sacristan A, Prieto D. Mechanisms of prostaglandin E1-induced relaxation in penile resistance arteries. J Urol. 2004 Feb;171(2 Pt 1):968-973.

31. Ruiz Rubio JL, Hernandez M, Rivera de los Arcos L, Benedito $\mathrm{S}$, Recio P, Garcia P, et al. Role of ATP-sensitive K+ channels in relaxation of penile resistance arteries. Urology. 2004Apr; 63 (4):800-805.

32. De los Arcos LR, Prieto D, Martinez AC, Benedito S, Hernandez M, Garcia-Sacristan A. An in vitro method of studying functional responses of penile resistance arteries under isobaric conditions. J Urol. 2004 May;171(5):1974-1978.

33. Prieto D, Rivera L, Recio P, Rubio JL, Hernandez M, GarciaSacristan A. Role of nitric oxide in the relaxation elicited by sildenafil in penile resistance arteries. J Urol. 2006 Mar; 175(3 Pt 1): 1164-1170.

34. Prieto D, Rivera L, Benedito S, Recio P, Villalba N, Hernandez $\mathrm{M}$, et al. $\mathrm{Ca} 2+$-activated $\mathrm{K}+(\mathrm{KCa})$ channels are involved in the relaxations elicited by sildenafil in penile resistance arteries. Eur J Pharmacol. 2006 Feb;15;531(1-3):232-237. Epub 2006 Jan 27.

35. Hernandez M, Barahona MV, Recio P, Benedito S, Martinez AC, Rivera L, et al. Neuronal and smooth muscle receptors involved in the PACAP- and VIP-induced relaxations of the pig urinary bladder neck. Br J Pharmacol. 2006 Sep;149(1):100-109. Epub 2006 Jul 17.

36. Hernández M, Recio P, Barahona MV, Bustamante S, Peña L, Martínez AC, et al. Pre-junctional a2-adrenoceptors modulation of the nitrergic transmission in the pig urinary bladder neck. Neurourol Urodyn. 2007;26(4):578-83.

Correspondencia autor: Dr. S. Bustamante Alarma Servicio Urología. Hospital Universitario Puerta de Hierro San Martín de Porres s/n - 28035 Madrid. Tel.: 913445000 E-mail autor: bustamantealarma@yahoo.es Información del artículo: Revisión - Experimentación básica Trabajo recibido: noviembre 2007

Trabajo aceptado: febrero 2008 